\title{
OPEN Recursive evolution of spin-wave multiplets in magnonic crystals of antidot-lattice fractals
}

\author{
Gyuyoung Park, Jaehak Yang \& Sang-Koog Kim ${ }^{\bowtie}$ \\ We explored spin-wave multiplets excited in a different type of magnonic crystal composed of \\ ferromagnetic antidot-lattice fractals, by means of micromagnetic simulations with a periodic \\ boundary condition. The modeling of antidot-lattice fractals was designed with a series of self-similar \\ antidot-lattices in an integer Hausdorff dimension. As the iteration level increased, multiple splits \\ of the edge and center modes of quantized spin-waves in the antidot-lattices were excited due to \\ the fractals' inhomogeneous and asymmetric internal magnetic fields. It was found that a recursive \\ development $\left(F_{n}=F_{n-1}+G_{n-1}\right)$ of geometrical fractals gives rise to the same recursive evolution of spin- \\ wave multiplets.
}

A recursive sequence is one of the most fundamental growth mechanisms in nature, interest in it having grown significantly for its potential quantum applications ${ }^{1-3}$. That is, a successive descendant of the nth generation is an aggregation of one or more preceding ascendants and their variations. More intriguing sequences are involved in fractal geometries in nature ${ }^{4}$. Those fractals are classified as statistical and random fractals ${ }^{5}$. Although the random fractals are relatively sporadic and weakly self-similar, the deterministic (exact) fractals have regularity and strong self-similarity. The recursive ordering has been discovered in the context of those two different fractal growths. Despite their close relationship, the recursive sequences in fractal growths have barely been studied in ordered spin systems.

Meanwhile, spatial periodicities of spin ordering in lattice crystals lead to the modifications of magnonic properties such as band structure ${ }^{6-8}$ and quantization ${ }^{9-12}$ of spin-waves. The antidot-lattice, a periodic array of many holes in a continuous film, has been a basic and promising two-dimensional (2D) magnonic crystal due to its scalability and good hysteric effect without superparamagnetic bottleneck ${ }^{13}$. The alteration of internal magnetic fields in the antidot-lattices results in several non-propagating eigenmodes even in forbidden bands. For example, edge modes, which resemble a "butterfly state"10, are localized around the boundary of each antidot, while center modes are extended along the channel in between the neighboring holes (antidots) ${ }^{12,14}$. Furthermore, standing spin-wave modes can be excited by different field conditions as well as in geometric confinements ${ }^{15}$. A variety of types of antidot-lattices have been employed that include bi-component (of different materials ${ }^{16}$ or different sizes of holes ${ }^{17}$ ), different Bravais type ${ }^{18}$, and defective lattices ${ }^{19}$. On the other hand, non-trivial magnonic dynamic behaviors were observed in aperiodic structures of antidots such as magnonic quasicrystals of Fibonacci structure ${ }^{20-22}$, Penrose and Ammann tilings ${ }^{23}$, and Sierpiński carpet ${ }^{24-26}$. In fact, Sierpiński fractals have led to unique phenomena in electronics ${ }^{27,28}$ and photonics ${ }^{29,30}$.

Model system of antidot-lattice fractals. Here, we propose magnonic crystals composed of ferromagnetic antidot-lattice fractals, arranged similarly to one of Sierpiński aperiodic motifs, as studied by micromagnetic simulations along with a delicate analysis of multiplet spin-wave modes. The overlap of scaled antidotlattices in a regular routine yields deterministic fractals, e.g., periodic structures with a local aperiodicity. This controllable non-statistical geometry provides a self-similarity in a part of the structure at every magnification, and can be designated according to the Hausdorff dimension of $\log _{S} \mathrm{~N}$, where $\mathrm{S}$ is the scale factor and $\mathrm{N}$ is the number of scaled objects ${ }^{31}$. That is, we used a series of scaled antidot-lattices (Fig. 1a) to construct antidot-lattice fractals deterministically with iterations, as illustrated in Fig. 1b. The antidot-lattices are self-similar in their geometric parameters of diameter $\mathrm{D}$ and lattice constant $\mathrm{L}$. The $\mathrm{D}_{\mathrm{n}}$ and $\mathrm{L}_{\mathrm{n}}$ of the nth antidot-lattice $\left(\mathrm{A}_{\mathrm{n}}\right)$ are exactly half of those of $A_{n-1}$. For example, $A_{2}$ has $L_{2}=L_{1} / 2$ and $D_{2}=D_{1} / 2$. Next, the nth fractal $S_{n}$ is constructed by the superposition of $A_{1}+A_{2}+\cdots+A_{n}$ as follows: $S_{1}=A_{1}, S_{2}=A_{1}+A_{2}, S_{3}=A_{1}+A_{2}+A_{3}$, and $S_{4}=A_{1}+A_{2}+A_{3}+A_{4}$,

National Creative Research Initiative Center for Spin Dynamics and Spin-Wave Devices, Nanospinics Laboratory, Research Institute of Advanced Materials, Department of Materials Science and Engineering, Seoul National University, Seoul 151-744, Republic of Korea. ${ }^{\circledR}$ email: sangkoog@snu.ac.kr 
(a)
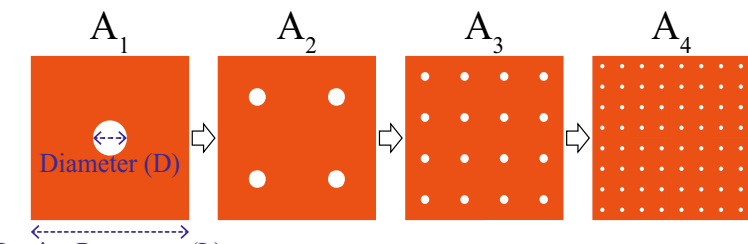

Lattice Parameter (L)

(b)

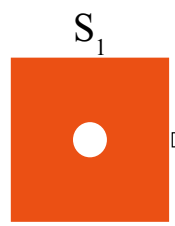

$\mathrm{A}_{1}$

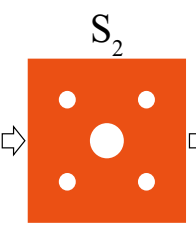

$\mathrm{A}_{1}+\mathrm{A}_{2}$

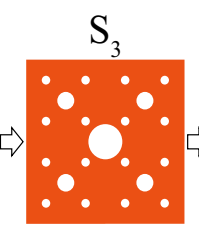

$\mathrm{A}_{1}+\mathrm{A}_{2}+\mathrm{A}_{3}$

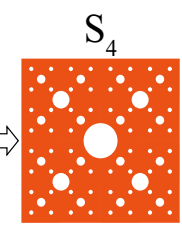

$\mathrm{A}_{1}+\mathrm{A}_{2}+\mathrm{A}_{3}+\mathrm{A}_{4}$

(c)
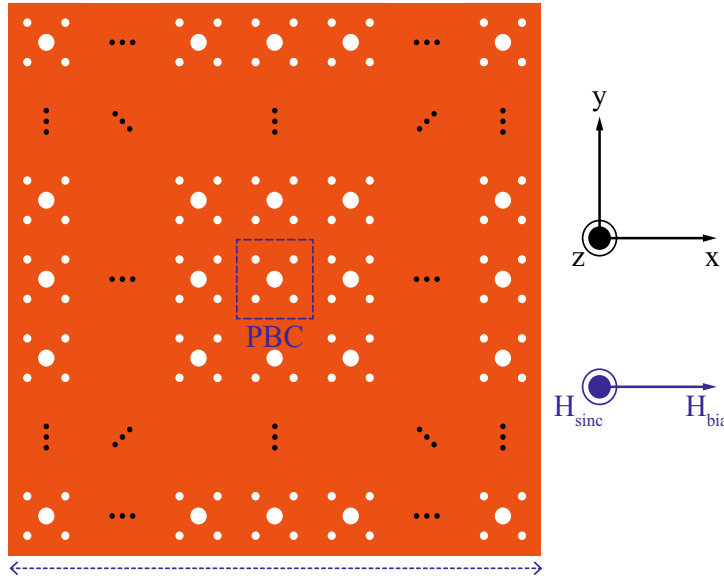

$100 \mu \mathrm{m}$

Figure 1. (a) Sequence of antidot-lattices with self-similar geometry. The capital letters of $\mathrm{D}$ and $\mathrm{L}$ correspond to the diameter of a hole and the size of a square Bravais lattice, respectively. (b) Evolution of antidot-lattice fractals. Each motif of $S_{n}$ denotes the superposition of the individual lattices of $A_{1}, A_{2}, \ldots$ and $A_{n}$. (c) Fractal magnonic crystal of $100 \mu \mathrm{m} \times 100 \mu \mathrm{m}$ dimensions where periodic boundary condition was applied using the area marked by blue-dashed box $\left(\mathrm{S}_{2}\right.$ as an example). For excitations of all possible spin-wave modes, dc-bias and sinc-function magnetic fields were applied in the $+\mathrm{x}$ direction and along the $\mathrm{z}$-axis, respectively.

as depicted in the series up to $S_{4}$ (see Fig. 1b). In detail, $A_{1}$ with $D=D_{1}$ and $L=L_{1}$ corresponds to the initiator (mother). $A_{1}$ and $A_{2}$ make up $S_{2}$. Since the $D_{2}$ and $L_{2}$ of $A_{2}$ are half those of $A_{1}$, the number of antidots for $A_{2}$ is increased by 4 times, and thus the Hausdorff dimension is $\log _{2} 4=2$. Following $A_{n}$ are scaled copies of previous $A_{n-1}$ in the same manner. Due to the self-similarity of the fractals, a recursive sequence arises inside the geometry of the motifs: let $\mathrm{F}_{\mathrm{n}}$ denote the geometrical sequence. $\mathrm{F}_{\mathrm{n}}(0 \leq x \leq L / 2)$ of each $\mathrm{S}_{\mathrm{n}}$ is a summation of $\mathrm{F}_{\mathrm{n}-1}(L / 4 \leq x \leq L / 2)$ and $\mathrm{G}_{\mathrm{n}-1}(0 \leq x<L / 4)$. The appearance of $\mathrm{F}_{\mathrm{n}-1}$ in $\mathrm{S}_{\mathrm{n}}$ is a scaled recursion of $\mathrm{F}_{\mathrm{n}-1}$ $(0 \leq x \leq L / 2)$ in $S_{n-1}$. In $S_{n}, G_{n-1}$ is a variation of $F_{n-1}$ and can be viewed as the $A_{1}$ antidot superimposed onto $\mathrm{F}_{\mathrm{n}-1}$.

Then, the 2D periodic lattice of magnonic crystals has a square Bravais symmetry, as shown in Fig. 1c.

\section{Results}

Recursive evolution of spin-wave multiplets. Figure 2 reveals that the spin-wave eigenmodes in the antidot-lattice fractals split into multiplets according to the recursive sequence (for better spectra, see also Supplementary Fig. S1). The first ordinary crystal denoted as $S_{1}\left(=A_{1}\right)$ exhibited three normal standing spin-wave modes as indexed by $\mathrm{E}_{1}, \mathrm{C}_{1}$, and $\mathrm{C}_{\mathrm{V} 1}{ }^{12}$. The very weak mode $\left(\mathrm{E}_{1}\right)$ at $1.77 \mathrm{GHz}$ corresponds to the edge mode, and the strongest mode $\left(\mathrm{C}_{1}\right)$ at $5.02 \mathrm{GHz}$ to the center mode. The two modes are periodically excited along the bias field direction. The last minor mode $\left(\mathrm{C}_{\mathrm{V} 1}\right)$ at $5.80 \mathrm{GHz}$ is a center-vertical mode (or a fundamental-localized mode) at the center between the neighboring antidots along the axis perpendicular to the bias field direction.

For $\mathrm{S}_{2}$, while keeping the $\mathrm{E}_{1}$ mode at a similar frequency, an additional doublet $\left(\mathrm{E}_{2}\right)$ of the edge mode appeared, which originated from $A_{2}$. The doublet $\left(C_{2}\right)$ of the center mode appeared as its substitution for the singlet $C_{1}$ in $\mathrm{S}_{1}$ (see Fig. 2b). The higher mode $(5.61 \mathrm{GHz})$ of the doublet $\mathrm{C}_{2}$ was hybridized with the $\mathrm{C}_{\mathrm{V} 1}$ mode $(5.80 \mathrm{GHz})$ in $S_{1}$. In a similar manner, for $S_{3}$, an additional triplet $\left(E_{3}\right)$ came from $A_{3}$, while the doublet $C_{2}$ in $S_{2}$ then became the triplet $\mathrm{C}_{3}$. The center-vertical mode $\left(\mathrm{C}_{\mathrm{V} 2}\right)$ of $\mathrm{A}_{2}$ was hybridized with the highest mode $(6.38 \mathrm{GHz})$ of $\mathrm{C}_{3}$. To 

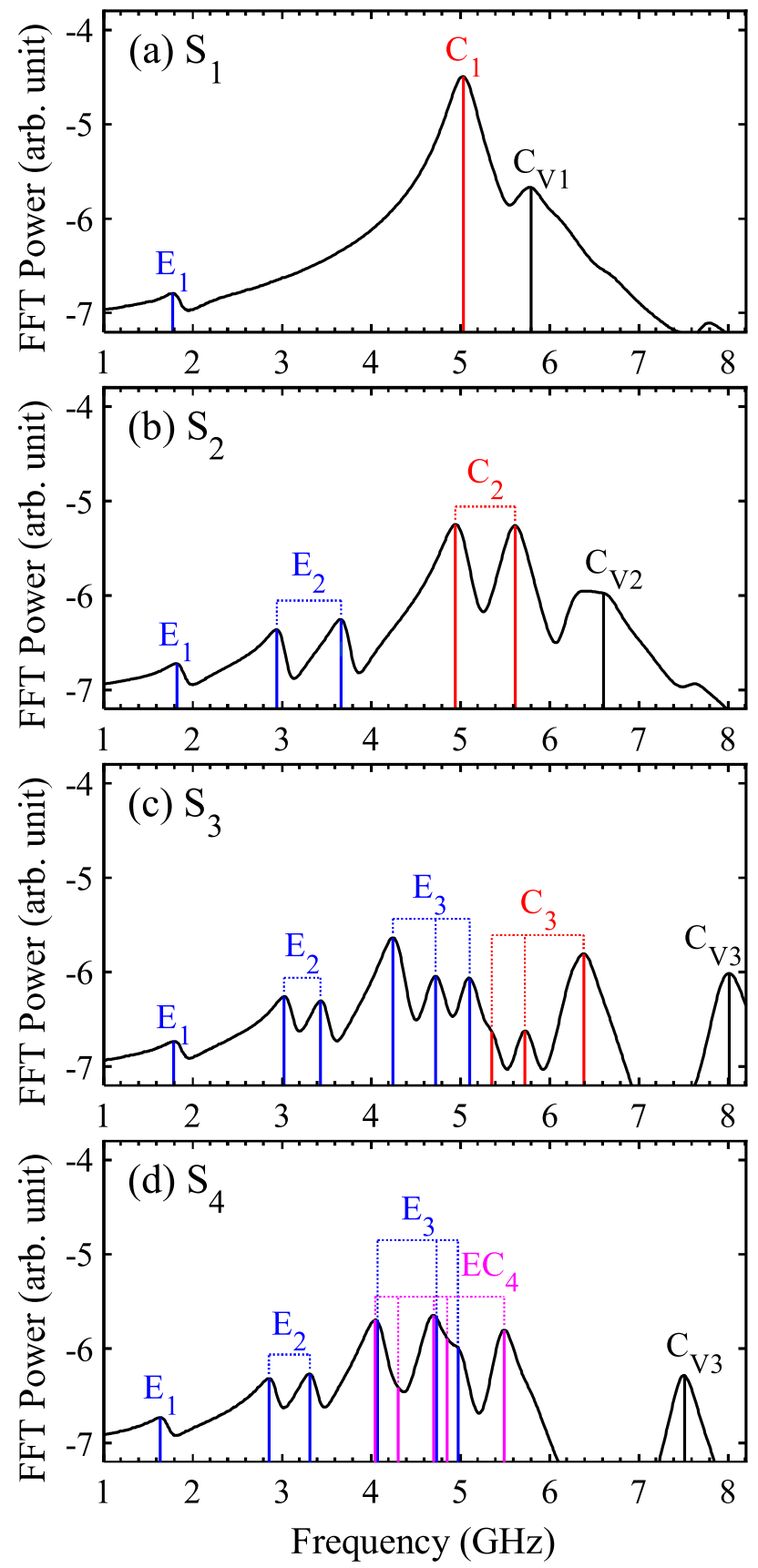

Figure 2. Modes' spectra in magnonic crystals of antidot-lattice fractals, $\mathrm{S}_{1}, \mathrm{~S}_{2}, \mathrm{~S}_{3}$, and $\mathrm{S}_{4}$, with $\mathrm{L}_{1}=1400 \mathrm{~nm}$ and $D_{1}=300 \mathrm{~nm} . E_{n}$ and $C_{n}$ denote the edge and center mode of $S_{n}$. A bias magnetic field of $30 \mathrm{mT}$ was applied in the $+\mathrm{x}$ direction.

sum up, by adding $A_{2}$ and $A_{3}$ to $S_{2}$ and $S_{3}$, respectively, each $E_{n}$ mode was newly updated while the $C_{n}$ mode substituted for $\mathrm{C}_{\mathrm{n}-1}$. This happened because the edge mode is strongly localized at the boundary of each antidot while the center mode is extended through the channels between the neighboring holes in the antidot-lattices. Whenever the next scaled antidot-lattices were overlapped to the previous one, each boundary of antidot entity of $A_{n}$ and $A_{n-1}$ remained intact with each other, while the channels of $A_{n}$ were impacted by the channels of $A_{n-1}$.

In the case of $\mathrm{A}_{4}$, the edge mode and the center mode were hybridized into one mode, because the hole-tohole distance was smaller than the previous antidot-lattices: the dipolar and exchange interactions were equally dominant at the narrow channel of $A_{4}$. Therefore, for $S_{4}$, the hybrid mode $\left(E_{4}+C_{4} \rightarrow E_{4}\right)$ appeared instead of the individual $\mathrm{E}_{4}$ and $\mathrm{C}_{4}$ modes. A total of five $\mathrm{EC}_{4}$ modes (pink-colored peaks) substituted for the $\mathrm{C}_{3}$ triplet.

The number of multiplets in the serial spectra followed the recursive sequence, $F_{n}=F_{n-1}+G_{n-1}$. The two eigenmodes $\left(E_{n}\right.$ and $\left.C_{n}\right)$ appeared as a singlet, doublet, triplet, and quintet for $S_{1}, S_{2}, S_{3}$ and $S_{4}$, respectively. Since there is only one zeroth $(n=0)$ standing spin-wave mode in unpatterned (continuous) thin film (i.e., $\left.S_{0}\right)$, the 


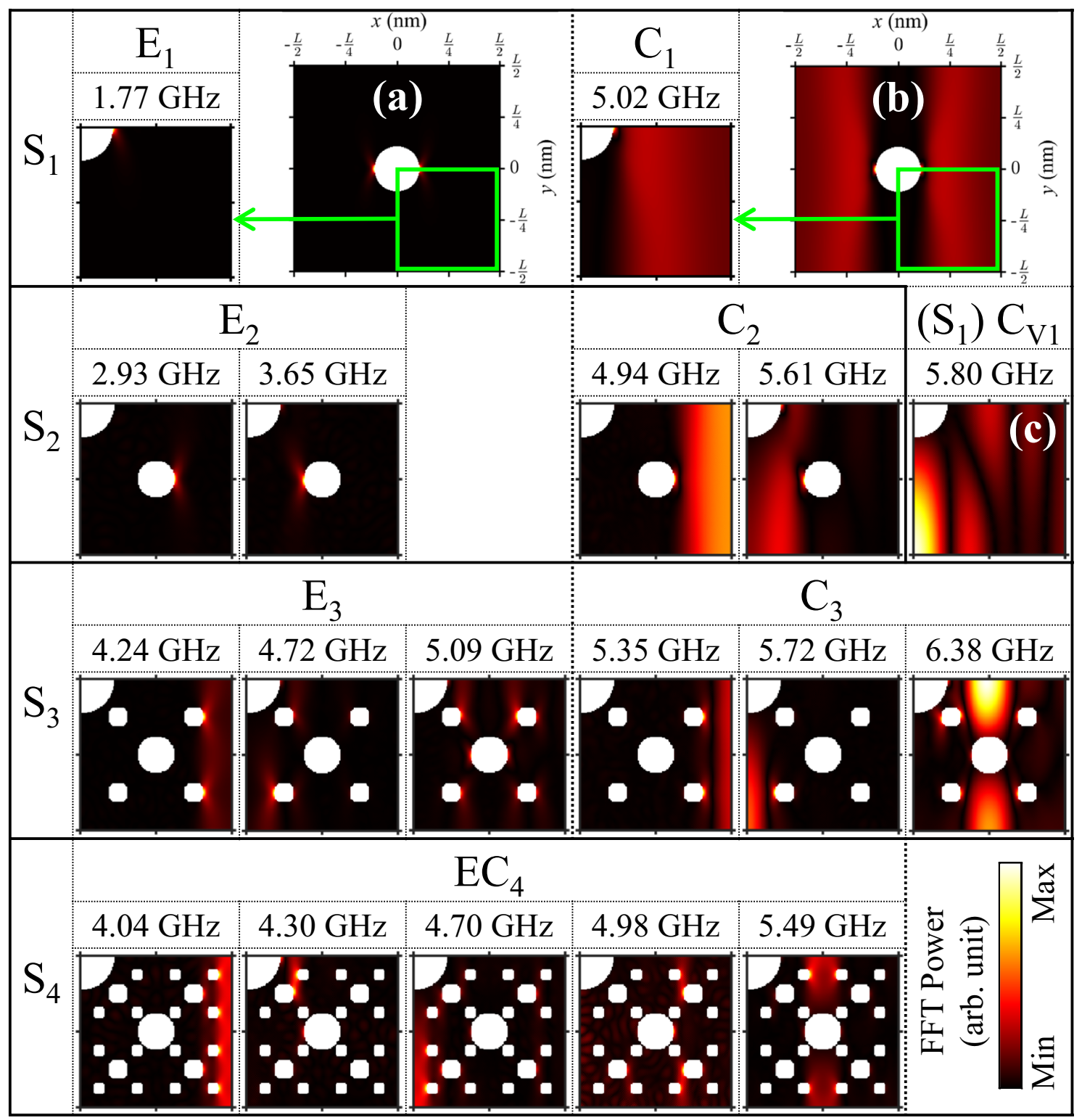

Figure 3. Spatial distribution of power of FFTs in bottom-right quarter area of motifs of $\mathrm{S}_{1}, \mathrm{~S}_{2}, \mathrm{~S}_{3}$ and $\mathrm{S}_{4}$ at indicated frequencies of specific modes.

number of the split modes corresponded to $1,1,2,3$, and 5 for $\mathrm{n}=0,1,2,3$, and 4, respectively, for both $\mathrm{E}_{\mathrm{n}}$ and $C_{n}$. The difference sequence $\left(G_{n}\right)$ is 1,1 , and 2 for $n=1,2$, and 3 , respectively.

In order to identify all of the excited modes represented by the FFT power-vs.-frequency spectra shown in Fig. 2, we performed FFTs on every single unit cell (or mesh) at the indicated resonance frequencies of the modes. Figure 3 shows the spatial distributions of FFT power in the bottom-right quarter of each motif $(0 \leq x \leq L / 2$, $-L / 2 \leq y \leq 0$ ) for each resonance frequency of the excited modes (for the corresponding phase profiles, see Supplementary Fig. S2). For $\mathrm{S}_{1}$, the major modes of $\mathrm{E}_{1}$ and $\mathrm{C}_{1}$ were visualized at 1.77 and $5.02 \mathrm{GHz}$, respectively. The edge mode was excited at the edge (or end) of the antidot (Fig. 3a), while the center mode was excited at the center (or channel) of the neighboring antidots (Fig. 3b). The higher mode $(5.61 \mathrm{GHz})$ of the $\mathrm{C}_{2}$ doublet, was vertically localized between the neighboring holes of $A_{1}$ in which $\mathrm{C}_{\mathrm{V} 1}$ was also localized in $\mathrm{S}_{1}$ (Fig. $3 \mathrm{c}$ ). This explains why the higher mode of $\mathrm{C}_{2}$ was hybridized with $\mathrm{C}_{\mathrm{V} 1}$, as mentioned earlier. On the other hand, the lower $(4.94 \mathrm{GHz})$ one of $\mathrm{C}_{2}$ remained extended along the channel between $\mathrm{S}_{2}$. The doublets of both $\mathrm{E}_{2}$ and $\mathrm{C}_{2}$ are antiphase with each other in temporal oscillation; $\mathrm{S}_{2}$ can be considered to be a two-dimensional nanooscillator. For $\mathrm{S}_{3}$, the highest $\mathrm{C}_{3}$ at $6.38 \mathrm{GHz}$ was fully localized in between the $\mathrm{A}_{2}$ antidots along the y-axis: it was hybridized with $\mathrm{C}_{\mathrm{V} 2}$ due to their shared localization area. The lowest $\mathrm{C}_{3}(5.35 \mathrm{GHz})$ remained extended along the channel between $\mathrm{S}_{3}$. Similarly, the lowest $\mathrm{EC}_{4}(4.04 \mathrm{GHz})$ for $\mathrm{S}_{4}$ was the only extended mode, while the others were localized in different local regions as noted by the red color. Since the distance between the antidots of $\mathrm{A}_{3}$ and $\mathrm{A}_{4}$ are close, some localized modes $(4.30 \mathrm{GHz}$ and $4.98 \mathrm{GHz})$ were excited at antidots of $\mathrm{A}_{3}$ together with certain antidots of $\mathrm{A}_{4}$. On the other hand, some of the $\mathrm{E}_{3}$ modes $(4.04 \mathrm{GHz}$ and $4.70 \mathrm{GHz})$ of $\mathrm{S}_{4}$ were excited at 


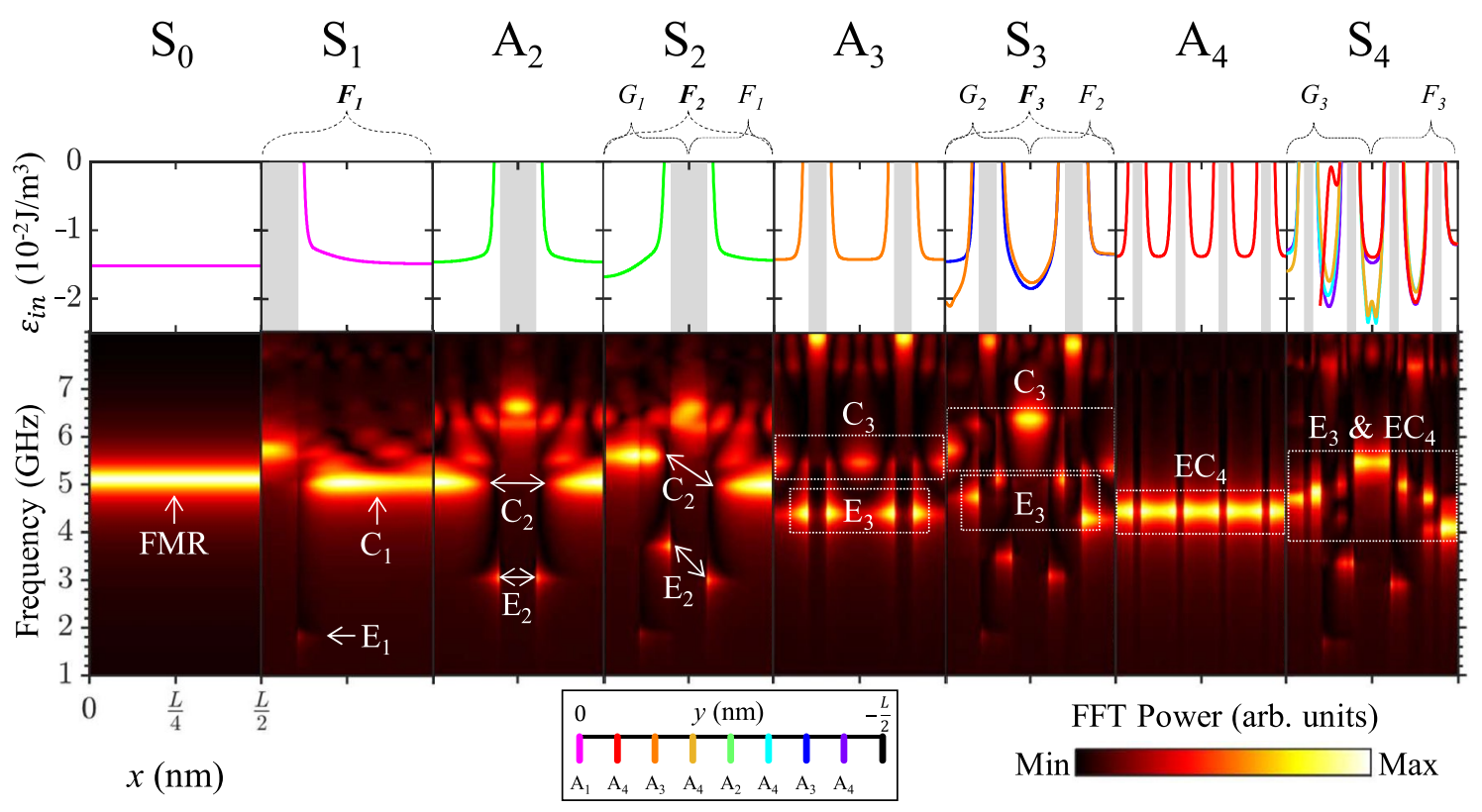

Figure 4. (Upper row) Spatial distributions of total magnetic energy density for thin film $\left(\mathrm{S}_{0}\right)$ and magnonic crystals of $A_{n}$ and $S_{n}$. The energy densities were plotted by cross-section along the y-axis where corresponding antidots were located. Each color of the plot matches with the index of the y-slice (black box) at the bottom of the figure. The gray-colored regions inside the plots denote the locations of the antidots. (Bottom row) Contour plots of FFT power on frequency and x position.

the same frequencies as those of the $\mathrm{EC}_{4}$ modes. Those $\mathrm{E}_{3}$ and $\mathrm{EC}_{4}$ modes become separated when the intensity of the bias field increased (see Supplementary Fig. S3).

The gap between the split modes narrowed down and finally merged into a singlet as the inhomogeneity of the magnetic energy decreased. In the other direction, the gap became wide and the corresponding different modes crossed over each other (showed conversion) as the inhomogeneity of the magnetic energy increased.

Similar to the recursive sequence in the geometrical fractals, we also found the recursive sequence in the evolution of the eigenmodes' spatial profiles, as shown in Fig. 4 . In detail, for the $E_{n}$ modes, let $E_{1}$ in $S_{1}$ be $F_{1}$. The two $\mathrm{E}_{2}$ modes in $\mathrm{S}_{2}$ are $\mathrm{F}_{2}$. The right part $(\mathrm{L} / 4 \leq \mathrm{x} \leq \mathrm{L} / 2)$ of the $\mathrm{E}_{2}(2.93 \mathrm{GHz})$ profile is the same as the $\mathrm{E}_{1}$ profile in $\mathrm{S}_{1} . \mathrm{E}_{2}(2.93 \mathrm{GHz})$ is $\mathrm{F}_{1}$ in $\mathrm{S}_{2}$. The left part $(0 \leq \mathrm{x}<\mathrm{L} / 4)$ of the $\mathrm{E}_{2}(3.65 \mathrm{GHz})$ profile is a variation of the $\mathrm{E}_{2}$ $(2.93 \mathrm{GHz})$ profile. $\mathrm{E}_{2}(3.65 \mathrm{GHz})$ is $\mathrm{G}_{1}$ in $\mathrm{S}_{2}$. The three $\mathrm{E}_{3}$ modes in $\mathrm{S}_{3}$ are $\mathrm{F}_{3}$. The right parts of the $\mathrm{E}_{3}(4.24 \mathrm{GHz}$ and $5.09 \mathrm{GHz}$ ) profiles are similar to the $\mathrm{E}_{2}$ profiles in $\mathrm{S}_{2}$. The two $\mathrm{E}_{3}$ modes are $\mathrm{F}_{2}$ in $\mathrm{S}_{3}$. The left part of the $\mathrm{E}_{3}$ $(4.72 \mathrm{GHz})$ profile is a variation of the $\mathrm{E}_{3}(4.24 \mathrm{GHz})$ profile. $\mathrm{E}_{3}(4.72 \mathrm{GHz})$ is $\mathrm{G}_{2}$ in $\mathrm{S}_{3}$. The five $\mathrm{EC}_{4}$ modes in $\mathrm{S}_{4}$ are $\mathrm{F}_{4}$. The right parts of the $\mathrm{EC}_{4}(4.04 \mathrm{GHz}, 4.98 \mathrm{GHz}$, and $5.49 \mathrm{GHz})$ profiles are similar to the $\mathrm{E}_{3}$ profiles in $\mathrm{S}_{3}$. The three $\mathrm{EC}_{4}$ modes are $\mathrm{F}_{3}$ in $\mathrm{S}_{4}$. The left parts of the $\mathrm{EC}_{4}(4.30 \mathrm{GHz}$ and $4.70 \mathrm{GHz})$ profiles are variations of the $\mathrm{EC}_{4}\left(4.98 \mathrm{GHz}\right.$ and $4.04 \mathrm{GHz}$, respectively) profiles. The two $\mathrm{EC}_{4}(4.30 \mathrm{GHz}$ and $4.70 \mathrm{GHz}) \mathrm{modes}$ are $\mathrm{G}_{3}$ in $\mathrm{S}_{4}$. In the same way, let $\mathrm{C}_{1}$ in $\mathrm{S}_{1}$ be $\mathrm{F}_{1}$. The two $\mathrm{C}_{2}$ modes in $\mathrm{S}_{2}$ are $\mathrm{F}_{2}$. The right part of the $\mathrm{C}_{2}(4.94 \mathrm{GHz})$ profile is the same as the $\mathrm{C}_{1}$ profile in $\mathrm{S}_{1}$. $\mathrm{C}_{2}(4.94 \mathrm{GHz})$ is $\mathrm{F}_{1}$ in $\mathrm{S}_{2}$. The left part of the $\mathrm{C}_{2}(5.61 \mathrm{GHz})$ profile is a variation of the $\mathrm{C}_{2}(4.94 \mathrm{GHz})$ profile. $\mathrm{C}_{2}(5.61 \mathrm{GHz})$ is $\mathrm{G}_{1}$ in $\mathrm{S}_{2}$. The three $\mathrm{C}_{3}$ modes in $\mathrm{S}_{3}$ are $\mathrm{F}_{3}$. The right parts of the $\mathrm{C}_{3}$ (5.35 GHz and $6.38 \mathrm{GHz}$ ) profiles are the same as the $\mathrm{C}_{2}$ profiles in $\mathrm{S}_{2}$. $\mathrm{C}_{3}\left(5.35 \mathrm{GHz}\right.$ and $6.38 \mathrm{GHz}$ ) are $\mathrm{F}_{2}$ in $\mathrm{S}_{3}$. The left part of the $\mathrm{C}_{3}(5.72 \mathrm{GHz})$ profile is a variation of the $\mathrm{C}_{3}(5.35 \mathrm{GHz})$ profile. $\mathrm{C}_{3}(5.72 \mathrm{GHz})$ is $\mathrm{G}_{2}$ in $\mathrm{S}_{3}$. The $\mathrm{EC}_{4}$ modes are considered in the same way as mentioned above.

Origin of recursive evolution. Next, in order to identify the splits of the spin-waves excited in $S_{n}$ with respect to $A_{n}$, we plotted the contours of FFT power for the frequency and the longitudinal x-direction, as shown in the bottom row of Fig. 4. In the upper row of Fig. 4, we also plotted the spatial distributions of the total magnetic energy density $\left(\mathcal{E}_{\text {tot }}\right)$, as expressed by

$$
\varepsilon_{\text {tot }}=-\frac{\mu_{0}}{2 V_{\text {mesh }}} \int \boldsymbol{M} \cdot\left(\boldsymbol{H}_{\text {Zeem }}+\boldsymbol{H}_{\text {demag }}\right) d V_{\text {mesh }}+A_{\text {exch }} \int|\nabla \boldsymbol{M}|^{2} d V_{\text {mesh }},
$$

where $\mathbf{H}_{\text {Zeem }}$ and $\mathbf{H}_{\text {demag }}$ are the Zeeman and demagnetization fields, respectively, $\mathbf{M}$ is the magnetization, $A_{\text {ex }}$ is the exchange constant, and $V_{\text {mesh }}$ is the volume of the mesh. The gray-colored regions depict the locations of the antidots inside each motif. The color of each plot matches with the y-slice index (the black box) at the bottom of Fig. 4. The FFT powers along the $\mathrm{x}$ distance $(0 \leq x \leq L / 2)$ agree well with the spatial distributions of the total energy density in terms of the $\mathrm{x}$ position. For example, the FMR mode was excited in the thin film (denoted as $\mathrm{S}_{0}$ ) at $5.10 \mathrm{GHz}$, as indicated by the homogeneous total energy distribution. The recursive sequence $\left(\mathrm{F}_{\mathrm{n}}\right)$ marked at the top of Fig. 4 denotes the evolution of both the total magnetic energy and the frequency of the eigenmodes. 
The right region $(L / 4 \leq x \leq L / 2)$ of $S_{n+1}$ is $F_{n}$. The appearance of $F_{n}$ in $S_{n+1}$ is similar to $F_{n}$ in $S_{n}$. The left region $(0 \leq x<L / 4)$ of $S_{n+1}$ is $G_{n}$, which is a variation of $F_{n}$ in $S_{n+1}$.

In $S_{1}$, at a similar frequency to that of the FMR mode, the $\mathrm{C}_{1}$ mode was excited at $5.02 \mathrm{GHz}$ in the region of $D / 2<x \leq L / 2$. To be specific, the FFT power spatially informed that the $\mathrm{C}_{1}$ mode started to be excited at the end of the $\mathrm{E}_{1}$ mode $(1.77 \mathrm{GHz})$ in terms of the $\mathrm{x}$ position. This profile well matches the total energy distribution of $S_{1}\left(=A_{1}\right)$. The magnetic energy variation inside the magnonic crystal corresponds to the localization of the quantized spin-wave modes. The mode arrangement of $A_{2}$ was similar to that of $A_{1}$. The $C_{2}$ mode of $A_{2}$ was excited at $5.03 \mathrm{GHz}$, whereas the $\mathrm{E}_{2}$ mode was excited at $3.00 \mathrm{GHz}$. With regard to $\mathrm{S}_{2}$, the $\mathrm{E}_{2}$ and $\mathrm{C}_{2}$ modes were split into doublets. Due to the existence of the $A_{1}$ antidot on the left side of the $A_{2}$ antidot, the energy profile on the left side is different from the right side of the $\mathrm{A}_{2}$ antidot. The $\mathrm{C}_{\mathrm{V} 1}$ mode and the shifted $\mathrm{C}_{2}$ mode were hybridized together, as shown in Fig. 3. In $\mathrm{S}_{3}$, the magnetic energy profile inside the motif was divided into three distinct regions. Therefore, the $\mathrm{E}_{3}$ mode at $4.42 \mathrm{GHz}$ in $\mathrm{A}_{3}$ became split into three modes at 4.24, 4.72, and 5.09 $\mathrm{GHz}_{\text {in }} \mathrm{S}_{3}$. Similarly, the $\mathrm{C}_{3}$ mode at $5.50 \mathrm{GHz}$ in $\mathrm{A}_{3}$ split into triplets $(5.35,5.72$, and $6.38 \mathrm{GHz})$ in $\mathrm{S}_{3}$. In $\mathrm{A}_{4}$, the $\mathrm{EC}_{4}$ mode was excited at $4.45 \mathrm{GHz}$, and the excitation profile showed that the edge and center modes had been hybridized into a single mode. Then, for $\mathrm{S}_{4}$, the $\mathrm{EC}_{4}$ mode was split into 5 modes (quintets). Since the $\mathrm{E}_{3}$ mode of $\mathrm{A}_{3}$ and the $\mathrm{EC}_{4}$ mode of $\mathrm{A}_{4}$ were excited at almost an equal frequency, a total of 8 split modes (the $\mathrm{E}_{3}$ triplets plus the $\mathrm{EC}_{4}$ quintets) in $\mathrm{S}_{4}$ were mixed up. The total energy distribution of $\mathrm{S}_{4}$ is complicated compared with those of the previous motifs, because of the complex arrangement of antidots inside $\mathrm{S}_{4}$.

The self-similarity of the fractal motifs introduces aperiodic arrangements of antidots in recursive order. In $S_{n}$, the total magnetic energy (most dominantly demagnetization energy) of the nth antidot array $\left(A_{n}\right)$ became aperiodic since the previous antidots modulated the magnetization configurations around $A_{n}$ antidots. The aperiodic energy variation inside the antidot-lattice fractals gives rise to the multiplets of the spin-wave eigenmodes under the recursive evolution. The energy aperiodicity can be reduced according to the geometric parameters or the externally applied magnetic fields in order to make the magnons' multiplets degenerate. As the dot-to-dot distance increased, the extent of aperiodicity decreased, and then the magnons' modes became degenerated (see Supplementary Fig. S5). In the same way, as the strength of the external magnetic field increased, the split modes were reunited (see Supplementary Fig. S3).

Origin of spin-wave multiplets. Finally, in order to examine the difference of the magnonic excitations between the fractal and non-fractal structures, we conducted the same simulation for the non-fractal, 2D type of $\mathrm{NaCl}$ lattice where two different radius holes are arranged alternately. This type of antidot-lattice has been studied under different terminologies, either composite-antidot array ${ }^{32,33}$ or bi-component antidot-lattice ${ }^{16,17}$. To avoid confusion, the term ' $2 \mathrm{D} \mathrm{NaCl}$ type' is employed to describe the antidot-lattice with alternating different diameters. The two antidot sublattices of $A_{1}$ and $A_{2}$ compose $S_{2}$ as well as $2 \mathrm{D} \mathrm{NaCl}$ type. In $S_{2}$, they satisfy the initiator-generator relationship with $\mathrm{L}_{2}=\mathrm{L}_{1} / 2$ and $\mathrm{D}_{2}=\mathrm{D}_{1} / 2$. In $2 \mathrm{D} \mathrm{NaCl}$ type geometry, both antidots exist in the $1: 1$ ratio, since $L_{2}=L_{1}$ but $D_{2}=D_{1} / 2$. The location of the $A_{1}$ antidot is asymmetric to that of the $A_{2}$ antidot in the $\mathrm{S}_{2}$ motif, while it is symmetric in the $2 \mathrm{D} \mathrm{NaCl}$ type motif. In both structures, antidots of $\mathrm{L}_{1}(\mathrm{~L})=1400 \mathrm{~nm}$ and $\mathrm{D}_{1}(\mathrm{D})=300 \mathrm{~nm}$ were used, while a $30 \mathrm{mT}$ strength of magnetic field was applied in the $+\mathrm{x}$-direction.

Figure 5 shows the FFT power versus frequency and the $\mathrm{x}$ position $(0 \leq x \leq L / 2)$ along with the total energy $\left(\varepsilon_{\text {tot }}\right)$ density distribution. In the range of $\mathrm{f}=1 \sim 6 \mathrm{GHz}$, the $\mathrm{E}_{1}, \mathrm{E}_{2}$ and $\mathrm{C}_{2}$ modes appeared noticeably in both $\mathrm{S}_{2}$ and $2 \mathrm{D} \mathrm{NaCl}$ type. Both of the $\mathrm{E}_{1}$ modes were independent singlets derived from the $\mathrm{A}_{1}$ antidots in both patterns. The only difference was that the $\mathrm{E}_{1}$ mode in $\mathrm{S}_{2}$ was excited at a slightly higher frequency, since the total magnetic field near the ends of the $A_{1}$ antidots in $S_{2}$ was higher than that of $2 \mathrm{D} \mathrm{NaCl}$ type. The $\mathrm{E}_{2}$ and $\mathrm{C}_{2}$ modes appeared as doublets only in $\mathrm{S}_{2}$, whereas those modes were typical singlets in $2 \mathrm{D} \mathrm{NaCl}$ type. In a comparison of the energy distributions between the two structures, the above difference resulted from the asymmetry of the internal energy about $x=L / 4$. Unlike $\mathrm{S}_{2}$, the non-fractal 2D NaCl type has the same energy distribution at both sides of the $\mathrm{A}_{2}$ hole; i.e., it shows a mirror symmetry about $x=L / 4$. For $\mathrm{S}_{2}$, the asymmetry of the total magnetic energy inside the fractal magnonic crystal is the origin of the spin-wave multiplets.

\section{Discussion}

The proposition of novel magnonic crystals composed of antidot-lattice fractals enlarges a basic understanding of quantized spin-wave modes. The fractals of 2-Hausdorff dimensions were constructed by the superposition of self-similar antidot-lattices. Local asymmetries inside the aperiodic magnonic motifs result in the split of the spin-wave eigenmodes: the edge mode, the center mode and the center-vertical mode (see Supplementary Fig. S5b,c). Due to the recursive sequence from the geometrical fractal growth, the local asymmetries inside the antidot-lattice fractals split the spin-waves into multiplets in the frequency spectra, showing the same recursive development. The split modes were finely localized into their own characteristic regions enabling selective excitation of the local area inside the magnonic crystals. Some of those split modes were reunited (or even duplicated) by the variations of the strength and direction of applied bias magnetic fields (see Supplementary Figs. S3 and S4, respectively). The reunion and the crossover among those finely divided modes would make the most of an active control with the bias magnetic field and the crystal geometry design (see Supplementary Fig. S5).

The proliferous standing spin-wave modes with fine localizations would be good candidates for magnonic devices that require diminutive excitation in a certain area of $2 \mathrm{D}$ nano-oscillators, memory devices, and sensors.

\section{Methods}

Micromagnetic simulation procedure. In the present simulations, we used an open-source software, $\operatorname{MuMax} 3^{34}$, which incorporates the Landau-Lifshitz-Gilbert equation ${ }^{35,36}$ along with GPU acceleration to solve the dynamic motions of individual magnetizations in given magnonic crystals, for example, as shown in Fig. 1c. 


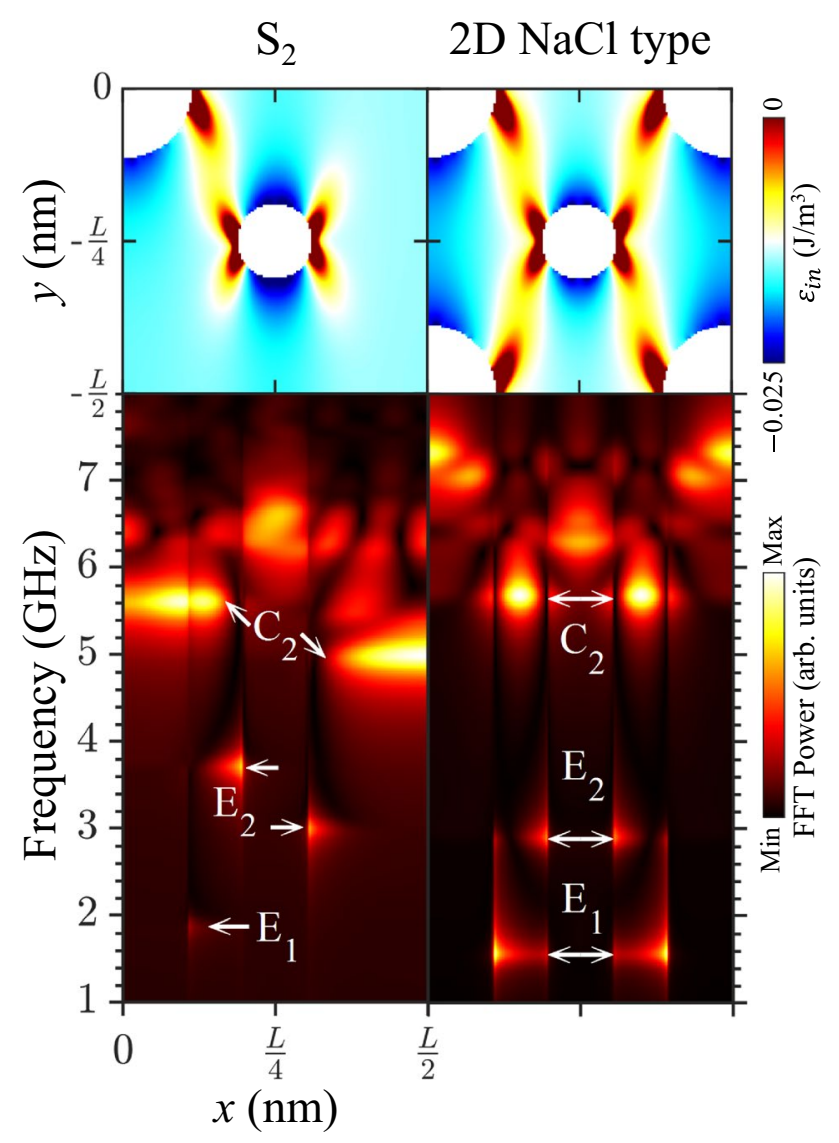

Figure 5. Comparison between $\mathrm{S}_{2}$ fractal and $2 \mathrm{D} \mathrm{NaCl}$ type (non-fractal) magnonic crystals: (Upper row) spatial distribution of total magnetic energy density in bottom-right quarter area of given motifs. (Bottom row) Spatial distribution of frequency spectra of FFT power in $\mathrm{x}$-direction. Doublets of $\mathrm{E}_{2}$ and $\mathrm{C}_{2}$ appeared only at the $\mathrm{S}_{2}$ fractal.

There, the motif, the $S_{2}$ fractal marked by a dashed square box, was extended to sufficiently large dimensions $(100 \mu \mathrm{m} \times 100 \mu \mathrm{m} \times 10 \mathrm{~nm})$ with a periodic boundary condition in order to avoid the distortions of the static and dynamic magnetizations at the discontinuous boundaries of its finite dimensions. The sizes of unit cells in the simulations were set up to $5 \mathrm{~nm} \times 5 \mathrm{~nm} \times 10 \mathrm{~nm}$. The material parameters used for Permalloy $\left(\mathrm{Py}: \mathrm{Ni}_{80} \mathrm{Fe}_{20}\right)$ were as follows: gyromagnetic ratio $\gamma=2.211 \times 10^{5}\left[\mathrm{~m} / \mathrm{A} \mathrm{s}\right.$ ], saturation magnetization $\mathrm{M}_{\mathrm{s}}=8.6 \times 10^{5}[\mathrm{~A} / \mathrm{m}]$, exchange stiffness $A_{\mathrm{ex}}=1.3 \times 10^{-11}[\mathrm{~J} / \mathrm{m}]$, damping constant $\alpha=0.01$, and zero magnetic anisotropy constant, $\mathrm{K}_{1}=\mathrm{K}_{2}=0$ $\left[\mathrm{J} / \mathrm{m}^{3}\right]$.

In order to excite spin-wave modes in the given magnonic crystals, we used a sinc (sine-cardinal) field as expressed by $\mathrm{h}(\mathrm{t})=\mathrm{h}_{0} \sin \left[2 \pi \mathrm{f}_{0}\left(\mathrm{t}-\mathrm{t}_{0}\right)\right] /\left[2 \pi \mathrm{f}_{0}\left(\mathrm{t}-\mathrm{t}_{0}\right)\right]$ with $\mu_{0} \mathrm{~h}_{0}=1 \mathrm{mT}, \mathrm{f}_{0}=20 \mathrm{GHz}, \mathrm{t}_{0}=1 \mathrm{~ns}$, and $\mathrm{t}=100 \mathrm{~ns}$. This pumping field was applied along the film normal under a dc bias field of $\mu_{0} \mathrm{H}_{\text {bias }}=30 \mathrm{mT}$ applied in the $+\mathrm{x}$ direction on the film plane (The eigenmodes of antidot-lattice fractals were stabilized at magnetic fields of greater strength than $20 \mathrm{mT}$; see Supplementary Fig. S3). The temporal oscillations of local magnetizations at each cell were transformed into the frequency domain via Fast Fourier Transforms (FFTs).

Received: 20 August 2021; Accepted: 11 October 2021

Published online: 19 November 2021

\section{References}

1. Möttönen, M., Vartiainen, J. J., Bergholm, V. \& Salomaa, M. M. Quantum circuits for general multiqubit gates. Phys. Rev. Lett. 93, 130502 (2004).

2. Flindt, C., Novotný, T., Braggio, A., Sassetti, M. \& Jauho, A.-P. Counting statistics of non-Markovian quantum stochastic processes. Phys. Rev. Lett. 100, 150601 (2008).

3. Hormozi, L., Zikos, G., Bonesteel, N. E. \& Simon, S. H. Topological quantum compiling. Phys. Rev. B 75, 165310 (2007).

4. Mitchison, G. J. Phyllotaxis and the Fibonacci series: An explanation is offered for the characteristic spiral leaf arrangement found in many plants. Science 196, 270-275 (1977).

5. Mandelbrot, B. B. Fractals: Form, Chance, and Dimension (W. H. Freeman \& Co., 1977).

6. Lee, K.-S., Han, D.-S. \& Kim, S.-K. Physical origin and generic control of magnonic band gaps of dipole-exchange spin waves in width-modulated nanostrip waveguides. Phys. Rev. Lett. 102, 127202 (2009). 
7. Kim, S.-K. Micromagnetic computer simulations of spin waves in nanometre-scale patterned magnetic elements. J. Phys. D: Appl. Phys. 43, 264004 (2010).

8. Jorzick, J. et al. Spin wave wells in nonellipsoidal micrometer size magnetic elements. Phys. Rev. Lett. 88, 047204 (2002).

9. Mruczkiewicz, M. et al. Standing spin waves in magnonic crystals. J. Appl. Phys. 113, 093908 (2013).

10. Martyanov, O. N. et al. Ferromagnetic resonance study of thin film antidot arrays: Experiment and micromagnetic simulations. Phys. Rev. B 75, 174429 (2007).

11. Yu, C., Pechan, M. J., Burgei, W. A. \& Mankey, G. J. Lateral standing spin waves in permalloy antidot arrays. J. Appl. Phys. 95, 6648 (2004).

12. Neusser, S., Botters, B. \& Grundler, D. Localization, confinement, and field-controlled propagation of spin waves in $\mathrm{Ni}_{80} \mathrm{Fe}_{20}$ antidot lattices. Phys. Rev. B 78, 054406 (2008).

13. Mandal, R. et al. Optically induced tunable magnetization dynamics in nanoscale Co antidot lattices. ACS Nano 6, 3397-3403 (2012).

14. Tacchi, S. et al. Universal dependence of the spin wave band structure on the geometrical characteristics of two-dimensional magnonic crystals. Sci. Rep. 5, 10367 (2015).

15. Vovk, A., Malkinski, L., Golub, V., Whittenburg, S. \& O’Connor, C. Preparation, structural characterization, and dynamic properties investigation of permalloy antidot arrays. J. Appl. Phys. 97, 10J506 (2005).

16. Tacchi, S. et al. Forbidden band gaps in the spin-wave spectrum of a two-dimensional bicomponent magnonic crystal. Phys. Rev. Lett. 109, 137202 (2012).

17. Tripathy, D., Vavassori, P., Porro, J. M., Adeyeye, A. O. \& Singh, N. Magnetization reversal and anisotropic magnetoresistance behavior in bicomponent antidot nanostructures. Appl. Phys. Lett. 97, 042512 (2010).

18. Krivoruchko, V. N. \& Marchenko, A. I. Apparent sixfold configurational anisotropy and spatial confinement of ferromagnetic resonances in hexagonal magnetic antidot lattices. J. Appl. Phys. 109, 083912 (2011).

19. Hu, X. K., Sievers, S., Müller, A. \& Schumacher, H. W. The influence of individual lattice defects on the domain structure in magnetic antidot lattices. J. Appl. Phys. 113, 103907 (2013).

20. Frotanpour, A. et al. Magnetization dynamics of a Fibonacci-distorted kagome artificial spin ice. Phys. Rev. B 102, 224435 (2020).

21. Lisiecki, F. et al. Magnons in a quasicrystal: propagation, extinction, and localization of spin waves in Fibonacci structures. Phys. Rev. Appl. 11, 054061 (2019).

22. Lisiecki, F. et al. Reprogrammability and scalability of magnonic Fibonacci quasicrystals. Phys. Rev. Appl. 11, 054003 (2019).

23. Watanabe, S., Bhat, V. S., Baumgaertl, K. \& Grundler, D. Direct observation of worm-like nanochannels and emergent magnon motifs in artificial ferromagnetic quasicrystals. Adv. Funct. Mater. 30, 2001388 (2020).

24. Swoboda, C., Martens, M. \& Meier, G. Control of spin-wave excitations in deterministic fractals. Phys. Rev. B 91, 064416 (2015).

25. Dai, Y. Y., Wang, H., Yang, T. \& Zhang, Z. D. Controlled magnetization reversal and magnetic spectra of artificial Sierpiński-fractal structure. J. Magn. Magn. Mater. 483, 70-75 (2019).

26. Barman, A., Mondal, S., Sahoo, S. \& De, A. Magnetization dynamics of nanoscale magnetic materials: A perspective. J. Appl. Phys. 128, $17901(2020)$.

27. Veen, E. V., Yuan, S., Katsnelson, M. I., Polini, M. \& Tomadin, A. Quantum transport in Sierpiński carpets. Phys. Rev. B 93, 115428 (2016).

28. Fremling, M., van Hoof, M., Smith, C. M. \& Fritz, L. Existence of robust edge currents in Sierpiński fractals. Phys. Rev. Res. 2, $013044(2020)$

29. De Nicola, F. et al. Multiband plasmonic Sierpiński carpet fractal antennas. ACS Photonics 5, 2418-2425 (2018).

30. Yang, Z., Lustig, E., Lumer, Y. \& Segev, M. Photonic Floquet topological insulators in a fractal lattice. Light Sci. Appl. 9, 128 (2020).

31. Hausdorff, F. Dimension und äußeres Maß. Math. Ann. 79, 157-179 (1918).

32. Vasseur, J. O., Dobrzynski, L., Djafari-Rouhani, B. \& Puszkarski, H. Magnon band structure of periodic composites. Phys. Rev. B 54, 1043 (1996).

33. Silhanek, A. V. et al. Enhanced vortex pinning by a composite antidot lattice in a superconducting Pb film. Phys. Rev. B 72, 014507 (2005).

34. Vansteenkiste, A. et al. The design and verification of MuMax3. AIP Adv. 4, 107133 (2014).

35. Landau, L. D. \& Lifshitz, E. M. On the theory of the dispersion of magnetic permeability in ferromagnetic bodies. Phys. Z. Sowjet. 8, 153-169 (1935).

36. Gilbert, T. L. A Lagrangian formulation of the gyromagnetic equation of the magnetization field. Phys. Rev. 100, 1243 (1955).

\section{Acknowledgements}

This research was supported by the Basic Science Research Program through the National Research Foundation of Korea (NRF) funded by the Ministry of Science, ICT and Future Planning (Grant No. I-AM35-21(041720210049)). The Institute of Engineering Research at Seoul National University provided additional research facilities for this work.

\section{Author contributions}

S.K.K. and G.P. conceived the main idea and the conceptual design of the experiments. G.P. performed the micromagnetic simulations. G.P., J.Y., and S.K.K. analyzed the data. S.K.K. and G.P. led the work and wrote the manuscript.

\section{Competing interests}

The authors declare no competing interests.

\section{Additional information}

Supplementary Information The online version contains supplementary material available at https://doi.org/ 10.1038/s41598-021-00417-0.

Correspondence and requests for materials should be addressed to S.-K.K.

Reprints and permissions information is available at www.nature.com/reprints.

Publisher's note Springer Nature remains neutral with regard to jurisdictional claims in published maps and institutional affiliations. 
(c) (i) Open Access This article is licensed under a Creative Commons Attribution 4.0 International cc) License, which permits use, sharing, adaptation, distribution and reproduction in any medium or format, as long as you give appropriate credit to the original author(s) and the source, provide a link to the Creative Commons licence, and indicate if changes were made. The images or other third party material in this article are included in the article's Creative Commons licence, unless indicated otherwise in a credit line to the material. If material is not included in the article's Creative Commons licence and your intended use is not permitted by statutory regulation or exceeds the permitted use, you will need to obtain permission directly from the copyright holder. To view a copy of this licence, visit http://creativecommons.org/licenses/by/4.0/.

(C) The Author(s) 2021 